\title{
Adaptação e implantação de um ERP open source em uma microempresa: Um estudo de caso prático
}

\author{
Luiz Arthur Melo dos Santos ${ }^{1}$, Jair da Silva Farias ${ }^{1}$, Lívia Maria Omena da Silva ${ }^{1}$ \\ ${ }^{1}$ Centro Universitário CESMAC \\ Rua Cônego Machado, 918, Farol, CEP: 57051-160 - Maceió/AL - Brasil \\ \{arthur.luiz, jair.farias, liviamariaomena\}@gmail.com
}

\begin{abstract}
This paper presents the adaptation and the beginning of the implementation of an open source ERP in a microenterprise from Alagoas, Brazil. The research and assessment of available systems were performed in order to guide the choice of the ERP most suitable to the needs of this company. OpenERP was chosen because it satisfied the microenterprise requirements, its superior usability compared to the other tested systems and its continuous improvements through the constant release of versions. The ERP adoption process started with the implementation of the warehouse management and the sales and purchases modules.
\end{abstract}

Resumo. Este artigo apresenta a adaptação e o início da implantação de um ERP open source em uma microempresa alagoana. Foi realizada uma pesquisa e avaliação de sistemas disponíveis com o intuito de guiar a escolha do ERP mais adequado às necessidades dessa microempresa. O ERP escolhido foi o OpenERP por atender aos requisitos da microempresa, além de sua usabilidade ser superior aos demais sistemas avaliados, sua evolução contínua através do lançamento constante de versões. Por fim, foi dado início ao uso do ERP com a implantação dos módulos de controle de estoque e registros de compras e vendas.

\section{Introdução}

Dados de outubro de 2012 apontam a existência de 4,4 milhões de micro e pequenas empresas no país, representando $99 \%$ das empresas privadas registradas no Brasil. [SEBRAE 2012]. Essas empresas empregam 52\% dos trabalhadores que possuem carteira assinada, gerando, aproximadamente, 15 milhões de empregos. [RAIS 2010 apud SEBRAE 2012]. As microempresas brasileiras têm, como uma de suas características, a baixa intensidade de capital, o que implica no baixo investimento em inovação tecnológica [IBGE 2003].

Uma das inovações que as empresas carecem é a Tecnologia da Informação (TI) - especificamente, o uso de sistemas de gestão empresarial. [CETIC 2012]. Esses sistemas, conhecidos como Enterprise Resource Planning (ERP), servem para informatizar e integrar os dados produzidos pelos diversos setores da empresa em um único local, possibilitando a sincronia de toda a sua cadeia de valor (da compra da matéria-prima à venda do produto final) e de suas atividades-meio (administração, finanças, marketing). [Perin 2010]. Essas informações são mantidas de forma 
organizada e acessível - podendo ser usadas, porventura, nas tomadas de decisões estratégicas pelos seus gestores.

O uso de sistemas ERP na gestão empresarial possibilita diversos benefícios: redução no fluxo de papéis e no tempo de obtenção e processamento de informações, melhoria no desempenho da empresa, redução de custos de mão de obra, melhoria do nível técnico dos funcionários, entre outros. [Mendes and Escrivão Filho 2003].

Segundo pesquisa realizada por [CETIC 2012], 71\% das empresas que possuem até 50 funcionários não utilizam uma ferramenta de ERP para auxiliar na gerência de seu negócio. Mesmo com a atenção das grandes empresas desenvolvedoras de sistemas ERP, o custo para a implantação continua alto para o orçamento dessas empresas, o que impede a realização de investimentos. [Souza and Saccol 2003] [Carvalho and Campos 2009].

Um sistema ERP open source tem, como uma de suas principais vantagens, a redução dos custos: tanto na sua adoção, quanto na sua adaptação aos processos e necessidades da empresa. [Carvalho and Campos 2009]. Não há cobrança de licença para uso. Sistemas ERP proprietários cobram pelo licenciamento para uso, assim como valores mensais para cada usuário. [Wailgum 2007].

A empresa B S Comércio Representações LTDA - ME (BSCR) gerencia seus processos essenciais (compra e venda de produtos, gerência de estoque, logística e finanças) de maneira manual. Isso implica em morosidade na execução de processos, menor eficiência e perda de competitividade. A manutenção de grande quantidade de documentos em papel requer considerável esforço, além disso, trata-se de material inflamável e sujeito à aceleração de seu processo natural de decomposição. Há dificuldades em processos de auditoria, identificação de problemas e/ou oportunidades de melhoria nos processos, entre outros.

Diante desse cenário, esta pesquisa aborda a seguinte questão: Como a empresa B S Comércio Representações LTDA - ME pode informatizar e integrar seus dados? A hipótese levantada é: O uso de um sistema ERP open source pode permitir a informatização e integração de dados, possibilitando a melhoria no controle gerencial, através da facilidade de acesso e transparência dos dados da empresa. Contudo, o objetivo dessa pesquisa é selecionar, analisar e implantar um sistema ERP open source que atenda às necessidades dessa empresa.

\section{Metodologia}

O levantamento de requisitos foi realizado através de entrevistas com o proprietário da empresa BSCR, a fim de se obter detalhes sobre processos e necessidades que precisam ser atendidas pelo sistema. Essas informações proporcionaram a definição dos critérios para escolha do ERP.

O processo de escolha foi embasado em testes de aceitação (através da instalação dos sistemas e análise de suas funcionalidades) e alinhado com pesquisas já realizadas por outros autores. [Tonini 2003] [Herzog 2006] [Fougatsaro 2009] [Gripe and Rodello 2011]. Entre os aspectos analisados, podem ser citados: o atendimento das necessidades da empresa, as funcionalidades já presentes no sistema, o suporte à legislação e ao 
sistema tributário brasileiro, a frequência de atualizações, o nível de documentação, a usabilidade (através de avaliação empírica com o proprietário da empresa), entre outros.

Após a escolha do sistema ERP a ser implantado na empresa, deu-se início ao estudo do sistema, análise das necessidades de adaptações e customizações, preparação da infraestrutura tecnológica e início da implantação de módulos do ERP para a empresa BSCR.

\section{Sistemas ERP open source}

Sistemas ERP são pacotes integrados de software, produzidos para dar suporte às operações das diversas áreas de negócio que fazem parte de uma empresa (suprimentos, manufatura, finanças, contabilidade, recursos humanos, etc.), mantendo as informações geradas na execução dessas operações em um repositório central de dados (banco de dados corporativo), possibilitando o reuso dessas informações pelos diversos setores da empresa [Zwicker and Souza 2003] [Fougatsaro 2009].

Um ERP é, geralmente, dividido em módulos que podem atender a um ou mais departamentos da empresa. São desenvolvidos de forma genérica, para que sejam capazes de atender ao maior número possível de empresas apenas com a parametrização do sistema (o preenchimento de campos variáveis, em tabelas do banco de dados, com informações específicas de cada empresa, sem a alteração do código fonte). [Zwicker and Souza 2003] [Carvalho and Campos 2009]. Apesar de ser apenas um software, sistemas ERP podem trazer consigo regras de negócio a serem seguidas pelas empresas que os adotam, podendo implicar mudanças estratégicas, organizacionais e culturais. [Davenport 1998] [Shehab, Sharp, Supramaniam and Spedding 2004 apud Fougatsaro 2009].

A implantação bem sucedida de um ERP pode trazer vários benefícios para a empresa. Sua adoção melhora a qualidade da informação, resultando em uma melhoria qualitativa no processo de tomada de decisão e no planejamento estratégico da empresa, criando oportunidades de inovação. [Saccol, Macadar, Pedron, Liberali Neto and Cazella 2003] [Lira, Parisi, Peleias and Peters 2012].

[Hunton, Lippincott and Reck 2003] compararam o desempenho de empresas que adotaram um ERP e com o de outras que não adotaram um sistema desse tipo. Os autores concluíram que, após três anos da implantação do sistema, aquelas que não adotaram tiveram um declínio significativo de desempenho em relação às empresas adotantes. [Zhang, Huang and Xu 2012] também observaram um aumento significativo no desempenho de empresas após o terceiro ano da implantação de um ERP.

Como a realização de adaptações de um sistema ERP às necessidades de uma empresa está inerente à sua implantação, as soluções ERP open source estão sendo cada vez mais aceitas no mercado [Serrano and Sarriegi 2006]. Esse tipo de sistema ERP é especialmente vantajoso para pequenas empresas, já que os custos com a infraestrutura necessária para seu funcionamento é baixo em relação ao de sistemas proprietários. Outra vantagem, proveniente do livre acesso ao código, é a independência em relação ao fornecedor de serviços ligados ao sistema, permitindo o desenvolvimento de novas funcionalidades e customizações internamente ou através de terceirizações [Carvalho and Campos 2009] [Fougatsaro 2009]. 


\section{Levantamento de requisitos e escolha do sistema a ser adotado}

A BSCR é uma empresa fundada em 1991, com atuação no mercado de distribuição de embalagens plásticas desde 1999. Além do foco em embalagens, a empresa também comercializa marmitas, papel e bebidas. A empresa encaixa-se na classificação de microempresa, tanto pelo seu porte quanto por seu faturamento. Emprega quatro funcionários, tendo apresentado um faturamento, em 2012, de aproximadamente R\$ 260.000,00 (duzentos e sessenta mil reais).

O proprietário da empresa é, também, gerente em uma empresa de grande porte do setor sucroalcooleiro, tendo experiência com processos informatizados através de um sistema ERP. No entanto, os demais funcionários da BSCR têm pouca ou nenhuma experiência com computadores.

O controle de estoque é um dos processos essenciais para a empresa, tendo em vista um universo de mais de 140 produtos ofertados, além da existência de dois locais de armazenamento e reservas de produtos nos próprios fornecedores. O proprietário reconhece o alto risco de falhas no registro manual de movimentações do estoque e sente a necessidade de seu controle mais refinado e efetivo.

Segundo o proprietário, a empresa necessita de uma ferramenta que permita o controle e a visualização do nível do estoque de diversos produtos vendidos, exibindo-o de forma organizada por suas características, fornecedores e/ou localização.

Atualmente, o relatório de fluxo de vendas é solicitado semanalmente. Os relatórios de movimentações do estoque e pagamentos recebidos são produzidos diariamente. Esses relatórios são gerados manualmente, algo trabalhoso, lento e sujeito a erros. O controle dos pagamentos recebidos é feito através de anotações em uma agenda do proprietário - o que, segundo ele, dificulta e retarda o uso dessas informações em consultas posteriores.

O proprietário busca, com a informatização dos dados da empresa, a criação de relatórios que forneçam as informações confiáveis e a consolidação dos registros de pagamentos recebidos, facilitando sua consulta e permitindo sua visualização sempre que se faça necessário. É de sua vontade, também, a obtenção de relatórios, a partir da ferramenta de ERP adotada, que permita a análise de índices de desempenho da empresa, especialmente os ligados à rentabilidade de produtos e clientes. Outro requisito é a possibilidade de acesso e atualização dos dados de operação da empresa através da Internet.

Por fim, após a estabilização do uso do sistema, o proprietário espera que o sistema seja capaz de integrar o registro de vendas com a geração de suas respectivas Notas Fiscais Eletrônicas (NF-e), permitindo que elas sejam emitidas a partir do próprio ERP.

\section{Análise dos sistemas ERP disponíveis}

Baseando-se nas citações de sistemas ERP open source feitas em outros estudos [Herzog 2006] [Serrano and Sarriegi 2006] [Correa 2008] [Carvalho and Campos 2009] [Fougatsaro 2009] [Eras, Fedichina, Namagatsu and Gozzi 2010] e, após buscas na 
Internet, foram selecionados seis sistemas para análise de sua adequação aos requisitos da empresa. São eles: FreedomERP, ERP5, Openbravo, Compiere, ADempiere e OpenERP.

Como apenas o FreedomERP é produzido por uma empresa brasileira, o principal aspecto analisado foi a adequação do sistema às obrigações fiscais e à legislação brasileira, processo denominado de localização do software [Correa 2008].

Analisando os sistemas ERP selecionados a partir das necessidades da empresa e o processo de localização dos sistemas produzidos por empresas estrangeiras, criou-se a Tabela 1, que sumariza o processo de filtragem das soluções encontradas para implantação.

Tabela 1. Análise de sistemas ERP open source.

\begin{tabular}{|c|c|c|c|c|c|c|c|}
\hline \multicolumn{8}{|c|}{ CRITÉRIOS DE AVALIAÇÃO } \\
\hline$\frac{\partial}{-1}$ & 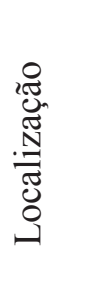 & 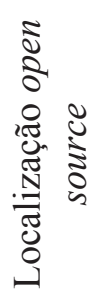 & 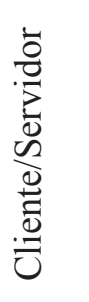 & 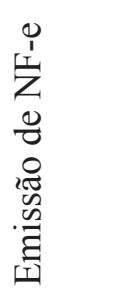 & 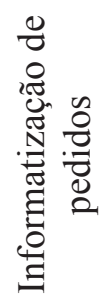 & 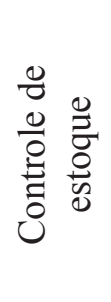 & 造 \\
\hline FreedomERP & Sim & Sim & Não & Não & Sim & Sim & Sim \\
\hline ERP5 & Não & Não & $\mathrm{Sim}$ & Não & Sim & Sim & Sim \\
\hline Openbravo & Sim & Não & Sim & Não & Sim & Sim & Sim \\
\hline Compiere & Sim & Não & Sim & Não & Sim & Sim & Sim \\
\hline ADempiere & Sim & Sim & $\mathrm{Sim}$ & Sim & Sim & Sim & Sim \\
\hline OpenERP & Sim & Sim & Sim & Parcial & Sim & Sim & Sim \\
\hline
\end{tabular}

Alguns dos critérios que foram considerados como não sendo atendidos pelos sistemas podem ter sido desenvolvidos por empresas e disponibilizados para seus clientes. No entanto, é importante ressaltar que, apesar de ser possível encontrar anúncios divulgando a disponibilidade dessas funcionalidades, não foi possível encontrá-las sob uma licença que siga o modelo open source, principal objetivo deste trabalho.

Analisando a tabela, observa-se que a informatização de pedidos, o controle de estoque e a geração de relatórios são funcionalidades básicas existentes em todos os sistemas, confirmando a exatidão na escolha de uma ferramenta ERP para atender às necessidades da empresa. Verifica-se, também, que apenas os sistemas ADempiere e OpenERP atendem a todas as necessidades da empresa, sobretudo fornecendo sua localização sob uma licença open source.

Apesar da parcialidade no atendimento da necessidade de emissão de NF-e pelo OpenERP, o sistema pode ser integrado a outras ferramentas que permitem a execução desse processo a contento. Pelo fato de existirem ferramentas open source com essa funcionalidade, o sistema não foi eliminado da seleção.

ADempiere ("cumprir", em italiano) foi um projeto criado em setembro de 2006, tendo como base o código-fonte do Compiere (prática denominada, pela comunidade 
open source, fork). O projeto possui membros em mais de 30 países, espalhados pelos cinco continentes, e tem como objetivo a criação e o suporte de soluções de negócio open source, através de um conjunto de aplicações de alta qualidade, que seja atrativa para parceiros fornecedores de suporte e com o máximo de participação de desenvolvedores. [Adempiere 2010].

OpenERP tem a visão de construir o melhor software de gerenciamento. Atualmente está disponível em 18 idiomas e com uma rede mundial de parceiros, com mais 800 desenvolvedores participando do projeto. O sistema é utilizado em grandes e pequenas empresas, de diversos setores econômicos, mostrando sua flexibilidade, capacidade de suprir as expectativas funcionais de seus usuários e facilidade de uso. [Fougatsaro 2009] [Pinckaers, Gardiner and Vossel 2011].

Após a realização da filtragem de sistemas ERP open source disponíveis que atendem às necessidades da empresa BSCR, passou-se à fase de testes desses sistemas para definição de qual seria adotado. Para a definição do sistema a ser implantado na BSCR, foram levados em consideração as categorias: frequência de atualizações e usabilidade, sugeridas por [Herzog 2006] [Fougatsaro 2009].

O ADempiere (e seu projeto de localização) utiliza o repositório Sourceforge, enquanto o OpenERP (assim como sua localização) utiliza o Launchpad. Ambos os repositórios, no entanto, fornecem informações sobre as últimas alterações e publicações do projeto, além da quantidade de membros que contribuem com seu desenvolvimento.

Segundo informações de [Sourceforge 2011] [ADempiere 2012], a última versão do ADempiere (370 LTS) foi lançada em $1^{\circ}$ de setembro de 2011 . No entanto, segundo [Santana 2012], a localização não é compatível com essa última versão, funcionando apenas com a versão 360 LTS do ADempiere - lançada em 2010. Já o OpenERP teve o lançamento de sua sétima versão em novembro de 2012, já havendo relatos da integração de sua localização à essa versão e também do uso do sistema em empresas. [Valyi 2013].

Para a comparação da usabilidade dos sistemas, realizou-se uma avaliação empírica com o proprietário da empresa, utilizando a observação direta intensiva (com a técnica de observação sistemática) para a determinação dos resultados [Marconi and Lakatos 2010]. Foi montado um ambiente de testes para os sistemas e solicitado, ao proprietário da empresa, a localização dos formulários de cadastro de clientes e de produtos.

Esse formato de teste segue modelo relatado por [Nielsen and Loranger 2007], que visa avaliar se é dado o suporte aos objetivos mais típicos de seus usuários. Segundo os autores, "essa é a maneira como a maioria dos estudos de usabilidade é conduzida e é excelente se você quiser descobrir como funcionam os elementos de design $[\ldots] "$.

Seguindo o modelo apresentado pelos autores, o proprietário da BSCR sentou-se em frente ao computador e o moderador atrás deste. Pediu-se para que o usuário pensasse em voz alta ao utilizar o sistema, o que, segundo os autores, serve para 
entender a razão de suas ações. Foram feitas duas gravações ${ }^{1}$, uma do monitor e outra do usuário, que incluem a gravação do áudio com seus comentários sobre a utilização do sistema - assim como prescrito pelos autores. A partir dessa avaliação empírica, foi possível determinar a superioridade de usabilidade do OpenERP. Os resultados são apresentados no quadro a seguir:

Quadro 1. Resultados do teste de usabilidade dos sistemas pré-selecionados.

\begin{tabular}{|c|c|}
\hline SISTEMA ERP & $\begin{array}{l}\text { TEMPO DE EXECUÇÃO DAS ATIVIDADES } \\
\text { PROPOSTAS }\end{array}$ \\
\hline ADempiere & $\begin{array}{l}\text { O usuário não conseguiu realizar nenhuma das duas tarefas } \\
\text { atribuídas em menos de } 10 \text { minutos. }\end{array}$ \\
\hline OpenERP & Cerca de 8 minutos para concluir as duas tarefas. \\
\hline
\end{tabular}

Além da consecução das tarefas dentro de um intervalo de tempo reduzido, os comentários feitos pelo proprietário da empresa, sobre sua percepção dos sistemas, também deixam claro a superioridade do OpenERP nesse quesito. Como os sistemas selecionados atendem vários dos critérios de avaliação expostos em outras pesquisas, a usabilidade tornou-se o principal aspecto a ser considerado para a definição do sistema a ser adotado.

Portanto, o OpenERP foi definido como o sistema a ser implantado na empresa por atender a todos os requisitos postos pela BSCR, além de ter demonstrado sua maior usabilidade - através das avaliações realizadas com o proprietário da empresa - e seu desenvolvimento ativo, observado através do lançamento constante e recente de novas versões.

\section{Processo de implantação do sistema}

Além da análise das necessidades de adaptações e customizações, outros passos iniciais da etapa de implantação foram executados durante a elaboração deste trabalho. Dentre os processos definidos por [Souza and Zwicker 2003], foi possível executar a escolha do modo de início de operação, a elaboração do planejamento geral de implantação e a preparação da infraestrutura tecnológica da empresa (juntamente com a criação de um ambiente de testes para o sistema), concluindo com a implantação de uma das funcionalidades solicitadas pela empresa.

Juntamente com o proprietário da empresa, definiu-se, inicialmente, o método conhecido por big-bang como modo de início de operação ideal para a implantação do sistema. Esse método prevê a instalação e customização de todos os módulos solicitados pela empresa, com a entrada em operação do sistema em uma mesma data. Essa decisão foi embasada em estudos [Mabert, Soni and Venkataramanan 2003] [Souza and Zwicker 2003] que indicam este método como o mais utilizado e adequado para empresas de menor porte, e também apontam o menor tempo para sua execução entre suas vantagens.

Para atingir o objetivo de implantar uma das funcionalidades solicitadas pela empresa, foram instalados os módulos de controle de estoque e a informatização do

\footnotetext{
1 As gravações dos testes de usabilidade estão disponíveis em:
} https://drive.google.com/folderview?id=0BzKW6EoXJjr9ZEdqT19HQTZVNUk\&usp=sharing 
registro de compras e vendas de produtos - levando, por consequência, à instalação de módulos para a manutenção de cadastros de produtos, de clientes e fornecedores e a instalação parcial da localização brasileira do sistema (especificamente, a ativação de campos para armazenar dados específicos do Brasil - CPF, Inscrição Estadual - e a importação de dados básicos para o uso do sistema, como estados, munícipios, instituições financeiras, entre outros).

Mesmo estando apenas em fase de homologação pelo proprietário, o processo de instalação desses módulos gerou novos conhecimentos das funcionalidades do sistema e de necessidades de adaptação - como previsto no modelo de ciclo de vida definido por [Souza and Zwicker 2000]. Uma das adaptações realizadas foi a tradução de alguns termos que não receberam tradução mesmo com a instalação parcial da localização essencial para o uso pleno do ERP, já que tanto o proprietário quanto seus funcionários não têm conhecimento de inglês.

Após a instalação dos módulos, realizou-se a importação dos dados já existentes. Como a empresa não possui grande parte de seus dados informatizados, não houve a possibilidade de criação de rotinas programadas para realizar a inserção desses dados no sistema.

Como exemplo, pode-se citar o processo de importação dos dados relativos ao estoque da empresa, realizado com base em informações de movimentação de estoque armazenadas em fichas de produto (pedaços de papel que mantêm um histórico de movimentação de produtos comercializados pela empresa, exibido na Imagem 1).

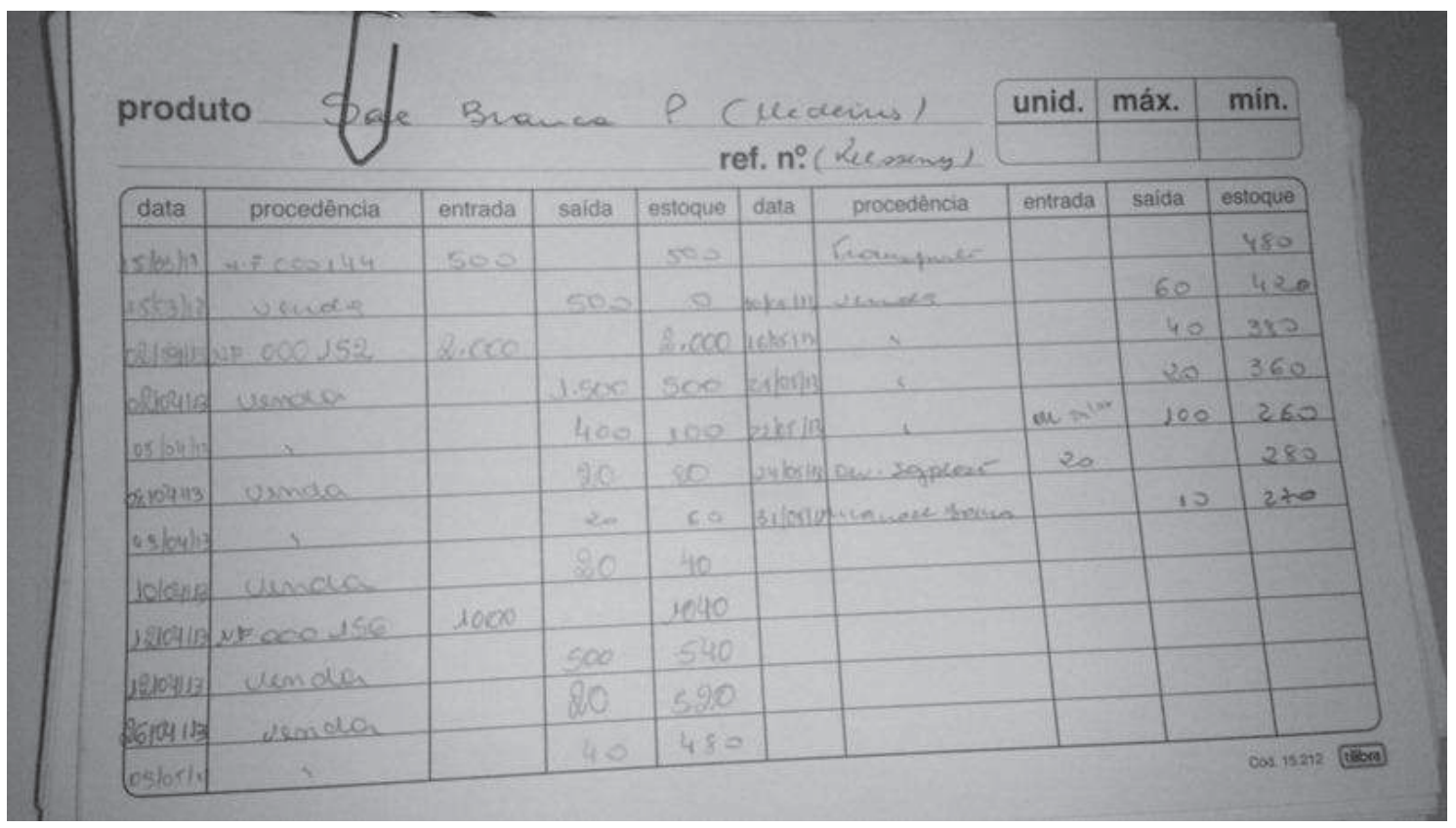

Imagem 1 - Ficha de produto utilizada para armazenar movimentações de estoque

A conclusão dessa etapa possibilitou a instalação e início da homologação da funcionalidade, referente à informatização do registro de pedidos - que se integra ao controle de movimentações do estoque -, possibilitando o fim do uso das fichas de produto para manter as informações sobre o estoque da empresa. 
Durante apresentação dos módulos instalados aos funcionários da BSCR, percebeu-se seu baixo nível de experiência em interação com computadores e conhecimentos de informática básica. Devido à escassez de tempo para o acompanhamento diário do uso do sistema por parte dos funcionários, acordou-se com o proprietário da BSCR a suspensão do processo de implantação de novos módulos. Tomou-se essa decisão pelo receio, por parte do proprietário, de paralisação do funcionamento da empresa e na tentativa de evitar alguns riscos da implantação de um sistema ERP já apontados por [Singh, Singh and Pereira 2010][Gabriel 2007], como a confusão em sua utilização por parte dos demais funcionários, a formação de resistências para seu uso e a entrada incorreta de dados.

Com a paralização da implantação do ERP e a falta de capacitação dos demais funcionários para utilizar o sistema, voltaram-se os esforços para o treinamento dos usuários que deverão utilizar o sistema. Como o treinamento dos usuários não está abarcado no escopo deste trabalho, seus resultados serão auferidos em trabalhos futuros.

\section{Considerações Finais e Próximos Passos}

Mesmo com a suspensão do processo de implantação de novos módulos do sistema, aqueles já instalados estão em pleno uso por parte do proprietário da empresa, tendo sido registradas mais de 5000 movimentações de estoque e cerca de 1300 vendas desde julho de 2013.

A partir de janeiro de 2014, deu-se início ao uso do módulo de controle de pagamentos das vendas, já tendo registrado mais de $\mathrm{R} \$ 600.000,00$ em faturas de clientes. A ativação desse módulo trouxe a possibilidade do proprietário obter relatórios relativos à rentabilidade de seus clientes e produtos, permitindo melhores decisões para ajustes de preços e para a política de descontos da empresa. Também foi possível realizar um melhor planejamento quanto à inadimplência de sua clientela, através de relatórios fornecidos pelo ERP indicando qual o prazo médio de atraso de pagamentos de cada cliente.

Apesar da realização de uma análise inicial das adaptações necessárias para a instalação da emissão de NF-e, não foi dado início ao processo de parametrização físcal do OpenERP, abrindo a possibilidade de realização de trabalhos futuros.

Outra possível pesquisa futura é quanto ao treinamento dos funcionários para o uso do sistema e a liberação de seu uso. A partir daí, seria apropriado realizar novos estudos sobre as etapas de estabilização e conclusão da implantação do ERP na empresa.

\subsection{Limitações da pesquisa}

O estudo de caso foi realizado para a produção de trabalho de conclusão de curso, o que gerou restrições quanto ao tempo e à necessidade de deslocamento para a sede da BSCR (que está localizada a cerca de 170 quilômetros de distância).

Outra limitação da pesquisa foi imposta pela constatação do baixo nível de conhecimento em informática dos funcionários da BSCR que deverão interagir com o sistema, o que impossibilitou a continuação do processo de parametrização e instalação de novos módulos, com a consequente liberação para homologação. 
A paralisação do processo de implantação do ERP também inviabilizou a análise da parametrização fiscal do sistema, para que, posteriormente, o proprietário da BSCR fosse capaz de emitir as NF-e a partir do próprio sistema.

\section{Referências}

ADEMPIERE. About ADempiere (2010). Disponível em: $<$ http://www.adempiere.com/What_does_ADempiere_mean $>$. Acesso em: 21 jan. 2014.

ADEMPIERE. News \& Updates - ADempiere ERP Wiki (2012). Acesso em: 21 jan. 2014.

CARVALHO, R. A. de and CAMPOS, R. de. (2009) "Uma análise de aspectos relacionados ao desenvolvimento e adoção de Enterprise Resources Planning livre de código aberto". Gestão \& Produção, São Carlos, v. 16, n. 4.

CETIC. Centro de Estudos sobre as Tecnologias da Informação e Comunicação. (2012) "Pesquisa sobre o uso das tecnologias da informação e comunicação no Brasil: TIC Domicílios e TIC Empresas” São Paulo: Comitê Gestor da Internet no Brasil.

CORREA, J. (2008) “Adoção, Seleção e Implementação de um ERP Livre”, Dissertação (Mestrado em Engenharia), Escola Politécnica da Universidade de São Paulo, Universidade de São Paulo, São Paulo.

DAVENPORT, T. H. (1998) "Putting the Enterprise into the Enterprise System". Harvard Business Review, Boston, Massachusetts: Harvard Business School Press.

ERAS, A. L., FEDICHINA, M. A. H., NAMAGATSU, F. A. and GOZZI, S. (2010) "Fatores competitivos na implementação de sistemas ERP open source: Um Estudo Comparativo entre fornecedor e usuários", In: Sustentabilidade Ambiental nas Organizações, XIII SEMEAD. São Paulo: USP.

FOUGATSARO, V. G. (2009) "A Study of Open Source ERP Systems”, Dissertação (Mestrado em Administração de negócios), School of Management, Blekinge Institute of Technology, Blekinge.

GABRIEL, A. E. P. A. (2007) "O papel do fornecedor de TI na transformação dos processos de negócio da pequena e média Empresa: um estudo de caso", Dissertação (Mestrado em Administração), Faculdade de Economia, Administração e Contabilidade, Universidade de São Paulo, São Paulo.

GRIPE, F. G. dos S. and RODELLO, I. A. (2011) "A theoretical analysis of key points when choosing open source ERP systems", JISTEM - Journal of Information Systems and Technology Management (Online), São Paulo, v. 8, n. 2.

HERZOG, T. (2006) “A Comparison of Open Source ERP Systems”, Viena: Vienna University of Technology.

HUNTON, J. E., LIPPINCOTT, B. and RECK, J. L. (2003) "Enterprise resource planning systems: comparing firm performance of adopters and nonadopters", International Journal of Accounting Information Systems, v. 4, n. 3, p. 165-184.

IBGE. Instituto Brasileiro de Geografia e Estatística. (2003) "As Micro e Pequenas Empresas Comerciais e de Serviços no Brasil: 2001”, Rio de Janeiro: IBGE. 
LIRA, A. M. de, PARISI, C., PELEIAS, I. R. and PETERS, M. R. S. (2012) "Uses of ERP systems and their influence on controllership functions in Brazilian Companies", JISTEM - Journal of Information Systems and Technology Management, São Paulo, v. 9, n. 2, ago.

MARCONI, M. de A. and LAKATOS, E. M. (2010) "Fundamentos de metodologia científica”, 7. ed. São Paulo: Atlas. ISBN 978-85-224-5758-8.

MENDES, J. V. and ESCRIVÃO FILHO, E. (2003) "Sistemas Integrados de Gestão (ERP) em Pequenas e Médias Empresas: Um Confronto entre a Teoria e a Prática Empresarial", In: SOUZA, C. A.; SACCOL, A. Z. (Org.). Sistemas ERP no Brasil: Teoria e Casos. 1 ed. São Paulo: Atlas. p. 243-265.

NIELSEN, J. and HORANGER, H. (2007) "Usabilidade na Web", Rio de Janeiro: Elsevier. ISBN 978-85-352-2190-9.

PERIN, E. (2010) "TI para Negócios: Como fazer a tecnologia trabalhar pelo seu sucesso e da sua empresa", 1. ed. São Paulo: Netpress Books. ISBN 978-85-63702$00-5$.

PINCKAERS, F., GARDINER, G. and VOSSEL, E. V. (2011) "OpenERP, a modern approach to integrated business management".

SACCOL, A. Z., MACADAR, M. A., PEDRON, C. D., LIBERALI NETO, G. and CAZELLA, S. C. (2003) "Sistemas ERP e seu Impacto sobre Variáveis Estratégicas de Grandes Empresas no Brasil", In: SOUZA, C. A.; SACCOL, A. Z. (Org.). Sistemas ERP no Brasil: Teoria e Casos. 1 ed. São Paulo: Atlas. p. 191-210.

SANTANA, R. A.. (2012) “ADempiere, ADempiereLBR \& Compiere • Exibir tópico Ajuda Adempiere 370LTS".

SEBRAE. Serviço Brasileiro de Apoio às Micro e Pequenas Empresas. (2012) "Boletim Estatístico das Micro e Pequenas Empresas".

SERRANO, N. and SARRIEGI, J. M. (2006) "Open Source ERPs: a new alternative for an old need", IEEE Software, Los Alamitos, v. 23, n. 3, p. 94-97, jun.

SINGH, L. P., SINGH, S. and PEREIRA, N. M. (2010) "Human risk factors in postimplementation phase of ERP in SMEs in India", In: Portland International Center for Management of Engineering and Technology, Proceedings Technology Management for Global Economic Growth. Portland, Oregon: PICMET, 2010.

SOURCEFORGE. (2011) “ADempiere ERP Business Suite - Browse”, ADempiere Official Release/Adempiere 3.7.0-LTS at SourceForge.net.

SOUZA, C. A. and SACCOL, A. Z. (2003) "Introdução", In: (Org.). Sistemas ERP no Brasil: Teoria e Casos. 1 ed. São Paulo: Atlas, p. 19-26.

SOUZA, C. A. de and ZWICKER, R. (2003) "Big-bang, small-bangs ou fases: estudo dos aspectos relacionados ao modo de início de operação de sistemas ERP”, Revista de Administração Contemporânea, Curitiba, v. 7, n. 4, dez.

TONINI, A. C. (2003) "Metodologia para Seleção de Sistemas ERP: Um Estudo de Caso”, In: SOUZA, C. A.; SACCOL, A. Z. (Org.). Sistemas ERP no Brasil: Teoria e Casos. 1 ed. São Paulo: Atlas, p. 29-60. 
VALYI, R. (2013) “Estabilidade Openerp 7 em ambiente de produção”, Google Groups. WAILGUM, T. (2007) “ERP Definitions and Solutions”, CIO, 07 mar.

ZHANG, L., HUANG, J. and XU, X. (2012 ) "Impact of ERP Investment on Company Performance: Evidence from Manufacturing Firms in China. Tsinghua Science And Technology, v. 17, n. 3, jun.

ZWICKER, R. and SOUZA, C. A. (2003) "Sistemas ERP: Conceituação, Ciclo de Vida e Estudos de Casos Comparados", In: SOUZA, C. A.; SACCOL, A. Z. (Org.). Sistemas ERP no Brasil: Teoria e Casos. 1 ed. São Paulo: Atlas, p. 63-87. 